\title{
ON THE OPTIMAL CONTROL FORCE APPLIED TO TUNED MASS DAMPERS FOR MULTI-DEGREE-OF-FREEDOM SYSTEMS
}

\author{
Nguren Dong AnH ${ }^{1}$ And Nguyen ChI SAng ${ }^{2}$ \\ ${ }^{1}$ Institute of Mechanics Hanoi \\ ${ }^{2}$ Research Institute of Mechanical Engineering
}

\begin{abstract}
The design of active TMD for multi-degree-of-freedom systems subjected to second order coloured noise excitation is considered using the linear quadratic optimal theory. A detailed numerical study is carried out for a 2-DOF system. It is shown that the effectiveness of active TMD is better than the one of passive TMD.
\end{abstract}

\section{Introduction}

Under environmental loading structures and machines may produce large undesired vibrations, which can reflect into their quality and durability. The use of tuned mass dampers (TMDs) helps to reduce the undesired vibrations in the primary systems. The passive TMDs [1-6] can only store or dissipate vibration energy, hence their application to the reduction of undesired vibrations is limited. This disadvantage of passive TMDs can be improved by applying to the passive TMDs an actuator force. In this case passive TMD becomes active TMD [7-11], which can change the system energy. In the paper [6] the design of optimal passive TMD for MDOF systems subjected to the second order coloured noise excitation has been investigated in order to minimize the sum of response mean square components of the primary system with a given ranking priority. In this paper an active TMD is considered based on the designed passive TMD. The main problem is to determine the optimal control force applied to the passive TMD.

\section{Second order coloured noise excitation}

The second order coloured noise process $p(t)$ is considered as a stationary response of the following filter of white noise

$$
\ddot{p}(t)+2 h \dot{p}(t)+\Omega^{2} p(t)=\sigma \dot{\xi}(t),
$$

where $h, \Omega, \sigma$ are positive constants, $\dot{\xi}(t)$ is the stationary Gaussian white noise process with unit intensity. The spectral density function of $p(t)$ is defined as

$$
S_{p}(\omega)=\frac{\sigma^{2}}{2 \pi\left[4 h^{2} \omega^{2}+\left(\Omega^{2}-\omega^{2}\right)^{2}\right]} .
$$

It is shown in [6] that the class of second order coloured noise processes (2.1) contains the white noise as well as narrow band processes. 


\section{Optimal active TMD}

The primary system consists of $n$ mass $m_{1}, m_{2}, \ldots, m_{n}$ put succeed one to another, each has its stiffness and damping coefficients denoted subsequently as $k_{1}, k_{2}, \ldots, k_{n}$; $c_{1}, c_{2}, \ldots, c_{n}$. The system is subjected to a ground excitation modeled by a second order coloured noise $p(t),(2.1)$. To suppress undesired large vibrations of the primary system, an optimal TMD of mass $m_{0}$ is connected to the mass $m_{n}$ (see Fig. 1). Suppose that the optimal values $k_{0}, c_{0}$ of the TMD are known according to the selection procedure proposed in $[6]$.

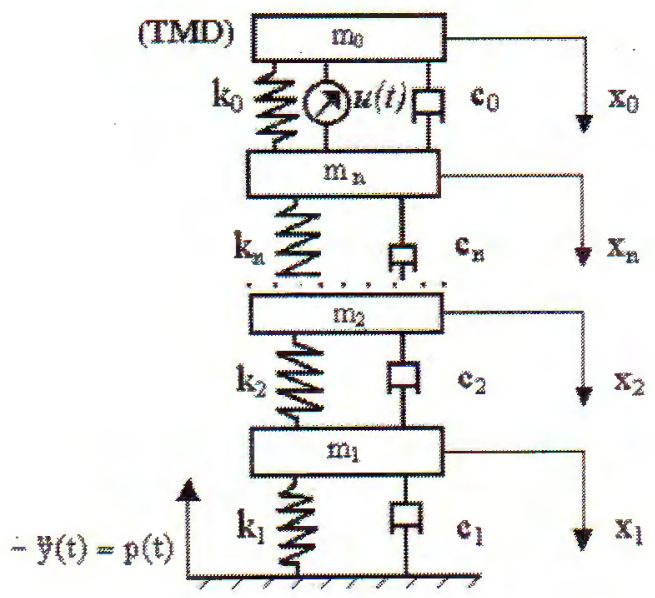

Fig. 1. System with active TMD

The passive TMD will become an active TMD if a control actuator force $u(t)$ based on the mass $m_{n}$ is applied to the passive TMD. The design problem of optimal active TMD is how to determine the optimal control force $u(t)$.

The equations of motion for the system with active TMD are described in the system of linear differential equations as follow

$$
M \ddot{X}(t)+C \dot{X}(t)+K X(t)=F(t)+E u(t),
$$

where

$$
\begin{aligned}
X(t) & =\left(x_{1}(t), x_{2}(t), \ldots, x_{n}(t), x_{0}(t)\right)^{T}, \\
M & =\left(\begin{array}{ccccc}
m_{1} & 0 & \cdots & 0 & 0 \\
0 & m_{2} & \cdots & 0 & 0 \\
\vdots & \vdots & \ddots & \vdots & \vdots \\
0 & 0 & \cdots & m_{n} & 0 \\
0 & 0 & \cdots & 0 & m_{0}
\end{array}\right),
\end{aligned}
$$




$$
\begin{aligned}
C & =\left[\begin{array}{ccccccc}
c_{1}+c_{2} & -c_{2} & 0 & \cdots & 0 & 0 & 0 \\
-c_{2} & c_{2}+c_{3} & -c_{3} & \cdots & 0 & 0 & 0 \\
0 & -c_{3} & c_{3}+c_{4} & \cdots & 0 & 0 & 0 \\
\vdots & \vdots & \vdots & \ddots & \vdots & \vdots & \vdots \\
0 & 0 & 0 & \cdots & c_{n-1}+c_{n} & -c_{n} & 0 \\
0 & 0 & 0 & \cdots & -c_{n} & c_{n}+c_{0} & -c_{0} \\
0 & 0 & 0 & \cdots & 0 & -c_{0} & c_{0}
\end{array}\right], \\
K & =\left[\begin{array}{ccccccc}
k_{1}+k_{2} & -k_{2} & 0 & \cdots & 0 & 0 & 0 \\
-k_{2} & k_{2}+k_{3} & -k_{3} & \cdots & 0 & 0 & 0 \\
0 & -k_{3} & k_{3}+k_{4} & \cdots & 0 & 0 & 0 \\
\vdots & \vdots & \vdots & \ddots & \vdots & \vdots & \vdots \\
0 & 0 & 0 & \cdots & k_{n-1}+k_{n} & -k_{n} & 0 \\
0 & 0 & 0 & \cdots & -k_{n} & k_{n}+k_{0} & -k_{0} \\
0 & 0 & 0 & \cdots & 0 & -k_{0} & k_{0}
\end{array}\right] .
\end{aligned}
$$

The base excitation force vector is

$$
F(t)=\left(p(t) m_{1}, p(t) m_{2}, \ldots, p(t) m_{n}, p(t) m_{0}\right)^{T} .
$$

The gain vector of control force is

$$
E=(0,0, \ldots, 1,-1)^{T} .
$$

Introduce the vector of state variables

$$
z(t)=\left(x_{1}(t), x_{2}(t), \ldots, x_{n}(t), x_{0}(t), \dot{x}_{1}(t), \dot{x}_{2}(t), \ldots, \dot{x}_{n}(t), \dot{x}_{0}(t)\right)^{T} .
$$

The equation of motion (3.1) can be rewritten in state variables as following

$$
\dot{z}=A z+B u(t)+H F(t),
$$

where the matrices $A_{(2 n+2) \times(2 n+2)}, H_{(2 n+2) \times(n+1)}$, vector $B_{(2 n+2) \times 1}$ take the form

$$
A=\left[\begin{array}{cc}
0 & I \\
-M^{-1} K & -M^{-1} C
\end{array}\right], \quad B=\left[\begin{array}{c}
0 \\
M^{-1} E
\end{array}\right], \quad H=\left[\begin{array}{c}
0 \\
M^{-1} I
\end{array}\right],
$$

where

$$
I=\operatorname{diagonal}(1,1, \ldots, 1), \quad \omega_{i}=\frac{k_{i}}{m_{i}}, \quad \xi_{i}=\frac{c_{i}}{2 m_{i} \omega_{i}}, \quad \mu_{i}=\frac{m_{i}}{m_{1}}, \quad i=0(1) n .
$$

Here, $\omega_{i}, \xi_{i}$ are the natural frequency, damping ratio of the separate mass $m_{i}$, respectively. The effectiveness of TMD again undesired vibrations can be improved by an adequate control force acting on the TMD. The objective of the study is how to define the optimal 
control force $u(t)$, which depends on the chosen control objectives. In the paper the following performance index of control is minimized

$$
J=\frac{1}{2}\left\langle\int_{0}^{t_{n}}\left[z^{T}(t) Q z(t)+\beta u^{2}(t)\right] d t\right\rangle \longrightarrow \min ,
$$

where $t_{n}$ is control time, $Q$ is a semi-positive weighting matrix, $\beta$ is a non-negative value. To solve the optimal control problem (3.8) subjected to the constrains represented by (3.6) one introduces firstly the Hamilton function with Lagrangian multiplier vector $\lambda(t)$

$$
H=\frac{1}{2} z^{T}(t) Q z(t)+\frac{1}{2} \beta u^{2}(t)+\lambda^{T}(A z+B u+H F-\dot{z}) .
$$

The necessary conditions for optimality are determined by the following equations

$$
\frac{\partial H}{\partial u}=0, \quad \dot{\lambda}^{T}=-\frac{\partial H}{\partial z},
$$

with boundary condition

$$
\lambda(T)=0
$$

Substituting (3.9) into (3.10) yields to differential equations

$$
\dot{\lambda}(t)=-A^{T} \lambda(t)-Q z(t)
$$

and the relation between the optimal control force and Lagrangian multiplier vector

$$
u(t)=-\frac{1}{\beta} B^{T} \lambda(t) .
$$

Using the feedback information on the system response

$$
\lambda(t)=S z(t)
$$

one can express the control force $u(t)$ in terms of system response

$$
u(t)=-\frac{1}{\beta} B^{T} S z(t)
$$

where the matrix $S(t)$ satisfies the Riccati equation

$$
S A+A^{T} S-\frac{1}{\beta} S B B^{T} S+Q=0 .
$$

Hence, the required control force $u(t)$ is determined by (3.15). The control system response is to be found from the systems of differential equations

$$
\dot{z}(t)=\left[A-\frac{1}{\beta} B B^{T} S(t)\right] z(t)+H F(t) .
$$




\section{Example of 2 DOF system}

In this section we investigate in more detail the effectiveness of active TMD for two-degreeof-freedom systems using numerical method. A model of 2-DOF system with active TMD is considered in Fig. 2. The active tuned mass damper $m_{0}$ here is connected to mass $m_{2}$ by a spring $k_{0}$, a damper $c_{0}$ and an applied control force $u(t)$.

The corresponding system of motion is given as follows

$$
\begin{aligned}
\dot{x}_{i} & =y_{i}, \\
\dot{y}_{i} & =g_{i}, \\
\dot{p} & =q, \\
\dot{q} & =-2 h q-\Omega^{2} p+\sigma \dot{\xi}(t), \\
& \quad i=1(1) 2,
\end{aligned}
$$

where

$$
\begin{aligned}
& g_{0}= \omega_{0}^{2} x_{2}-\omega_{0}^{2} x_{0}+2 \omega_{0} \xi_{0} y_{2}-2 \omega_{0} \xi_{0} y_{0}-\frac{1}{m_{0}} u(t)+p(t), \\
& g_{1}=\left(-\omega_{1}^{2}-\mu_{2} \omega_{2}^{2}\right) x_{1}+\mu_{2} \omega_{2}^{2} x_{2}+\left(-2 \omega_{1} \xi_{1}-2 \mu_{2} \xi_{2} \omega_{2}\right) y_{1}+2 \mu_{2} \xi_{2} \omega_{2} y_{2}+p(t), \\
& g_{2}=\omega_{2}^{2} x_{1}+\left(-\omega_{2}^{2}-\frac{\mu_{0}}{\mu_{2}} \omega_{0}^{2}\right) x_{2}+\frac{\mu_{0}}{\mu_{2}} \omega_{0}^{2} x_{0}+2 \xi_{2} \omega_{2} y_{1} \\
&+\left(-2 \xi_{2} \omega_{2}-2 \frac{\mu_{0}}{\mu_{2}} \xi_{0} \omega_{0}\right) y_{2}+2 \frac{\mu_{0}}{\mu_{2}} \xi_{0} \omega_{0} y_{0}+\frac{1}{m_{2}} u(t)+p(t) . \\
& \text { (TMD) }
\end{aligned}
$$

Fig. 2. Model of 2-DOF system with active TMD

For numerical study the following values of system parameters are considered

$$
\omega_{1}=40, \quad \omega_{2}=50, \quad \xi_{1}=0.25, \quad \xi_{2}=0.1, \quad \mu_{2}=0.3, \quad m_{1}=1
$$

and the excitation parameters as

$$
h=2, \quad \Omega=30, \quad \sigma=1 .
$$

Given values (4.3) and (4.4) the following parameters of passive TMD can be found using the selection procedure proposed in [6]:

$$
\mu_{0}=0.04, \quad \omega_{0}=29.25, \quad \xi_{0}=0.026 .
$$


Determine now the optimal control force $u(t)$. For that purpose one solves the Riccati equation (3.16) where

$$
A=\left[\begin{array}{cccccc}
0 & 0 & 0 & 1 & 0 & 0 \\
0 & 0 & 0 & 0 & 1 & 0 \\
0 & 0 & 0 & 0 & 0 & 1 \\
-2350 & 750 & 0 & -23 & 3 & 0 \\
2500 & -2614.075 & 114.075 & 10 & -10.2028 & 0.2028 \\
0 & 855.5625 & -855.5625 & 0 & 1.521 & -1.521
\end{array}\right], B=\left[\begin{array}{c}
0 \\
0 \\
0 \\
0 \\
3.333333 \\
-25
\end{array}\right]
$$

$$
\beta=10^{-6}, \quad Q=\left[\begin{array}{cccccc}
1 & 0 & 0 & 0 & 0 & 0 \\
0 & 1 & 0 & 0 & 0 & 0 \\
0 & 0 & 0 & 0 & 0 & 0 \\
0 & 0 & 0 & 0 & 0 & 0 \\
0 & 0 & 0 & 0 & 0 & 0 \\
0 & 0 & 0 & 0 & 0 & 0
\end{array}\right]
$$

\subsection{Numerical calculation}

Using Matlab R12 one has

$$
\begin{aligned}
& S=\left[\begin{array}{ccc}
0.03184132444405 & 0.00097724714208 & 0.00050570471817 \\
0.00097724714208 & 0.01971750713149 & -0.00005171977745 \\
0.00050570471817 & -0.00005171977745 & 0.00006762830833 \\
0.00040555100811 & -0.00006183295253 & 0.00001957380918 \\
0.00018990837907 & 0.00007189707920 & 0.00001554588380 \\
0.00001698304480 & -0.00001868091846 & 0.00000207278451
\end{array}\right. \\
& \left.\begin{array}{llll}
0.00040555100811 & 0.00018990837907 & 0.00001698304480
\end{array}\right] \\
& \begin{array}{llll}
-0.00006183295253 & 0.00007189707920 & -0.00001868091846
\end{array} \\
& \begin{array}{lll}
0.00001957380918 & 0.00001554588380 & 0.00000207278451
\end{array} \\
& \begin{array}{lll}
0.00002086465134 & 0.00000933367450 & 0.00000149107150
\end{array} \\
& \begin{array}{llll}
0.00000933367450 & 0.00000902250590 & 0.00000102948081
\end{array} \\
& \left.\begin{array}{llll}
0.00000149107150 & 0.00000102948081 & 0.00000021630954
\end{array}\right] \\
& \begin{array}{r}
G=10^{2}\left[\begin{array}{ccc}
2.08451810241633 & 7.06679921167735 & -0.00000000005156 \\
-0.06164539266206 & 0.04337999496807 & -0.01976135826795
\end{array}\right],
\end{array}
\end{aligned}
$$

where $S$ is Riccati matrix, $G$ is gain matrix.

$$
G=\frac{1}{\beta} B^{T} S .
$$

The control force is

$$
u=-G z,
$$


where $z=\left[\begin{array}{llllll}x_{1} & x_{2} & x_{0} & y_{1} & y_{2} & y_{0}\end{array}\right]$.

To estimate the effectiveness of passive and active TMD one can use ratios $k_{P}$ and $k_{A}$ defined as

$$
k_{P i}=\frac{\left\langle x_{P i}^{2}\right\rangle}{\left\langle x_{0 i}^{2}\right\rangle}, \quad k_{A i}=\frac{\left\langle x_{A i}^{2}\right\rangle}{\left\langle x_{0 i}^{2}\right\rangle}, \quad(i=1(1) 2)
$$

where $\left\langle x_{0 i}^{2}\right\rangle,\left\langle x_{P i}^{2}\right\rangle,\left\langle x_{A i}^{2}\right\rangle$ are the mean squares of $x_{i}(i=1(1) 2)$ in three cases: without TMD, with passive TMD and with active TMD, respectively. These mean squares can be obtained by using equation system (4.1) to form following moment equations [4]

$$
\sum_{n=1}^{n}\left[\left\langle y_{i} \frac{\partial \varphi}{\partial x_{i}}\right\rangle+\left\langle g_{i}(\ldots) \frac{\partial \varphi}{\partial y_{i}}\right\rangle\right]+\left\langle q \frac{\partial \varphi}{\partial p}\right\rangle+\left\langle\left(-2 h q-\Omega^{2} p\right) \frac{\partial \varphi}{\partial q}\right\rangle+\frac{1}{2} \sigma^{2}\left\langle\frac{\partial^{2} \varphi}{\partial^{2} q}\right\rangle=0,
$$

where $\langle\ldots\rangle$ is the mathematical expectation and the functions are selected as follow

$$
\begin{array}{llllll}
\varphi_{0}=x_{0}^{2}, & \varphi_{1}=x_{1}^{2}, & \varphi_{2}=x_{2}^{2}, & \varphi_{3}=y_{0}^{2}, & \varphi_{4}=y_{1}^{2}, & \varphi_{5}=y_{2}^{2}, \\
\varphi_{6}=x_{0} x_{1}, & \varphi_{7}=x_{0} x_{2}, & \varphi_{8}=x_{0} y_{0}, & \varphi_{9}=x_{0} y_{1}, & \varphi_{10}=x_{0} y_{2}, & \varphi_{11}=x_{0} p, \\
\varphi_{12}=x_{0} q, & \varphi_{13}=x_{1} x_{2}, & \varphi_{14}=x_{1} y_{0}, & \varphi_{15}=x_{1} y_{1}, & \varphi_{16}=x_{1} y_{2}, & \varphi_{17}=x_{1} p, \\
\varphi_{18}=x_{1} q, & \varphi_{19}=x_{2} y_{0}, & \varphi_{20}=x_{2} y_{1}, & \varphi_{21}=x_{2} y_{2}, & \varphi_{22}=x_{2} p, & \varphi_{23}=x_{2} q, \\
\varphi_{24}=y_{0} y_{1}, & \varphi_{25}=y_{0} y_{2}, & \varphi_{26}=y_{0} p, & \varphi_{27}=y_{0} q, & \varphi_{28}=y_{1} y_{2}, & \varphi_{29}=y_{1} p, \\
\varphi_{30}=y_{1} q, & \varphi_{31}=y_{2} p, & \varphi_{32}=y_{2} p, & \varphi_{33}=p^{2}, & \varphi_{34}=q^{2}, & \varphi_{35}=p q .
\end{array}
$$

Numerical calculated results are put into Table 1 . The mean squares of $x_{1}, x_{2}$, control force $u(t)$ and base excitation force $p(t)$ are calculated in three cases: without TMD, passive TMD and active TMD. The effectiveness of active TMD is much better than the one of passive TMD and the control force is smaller than base excitation force.

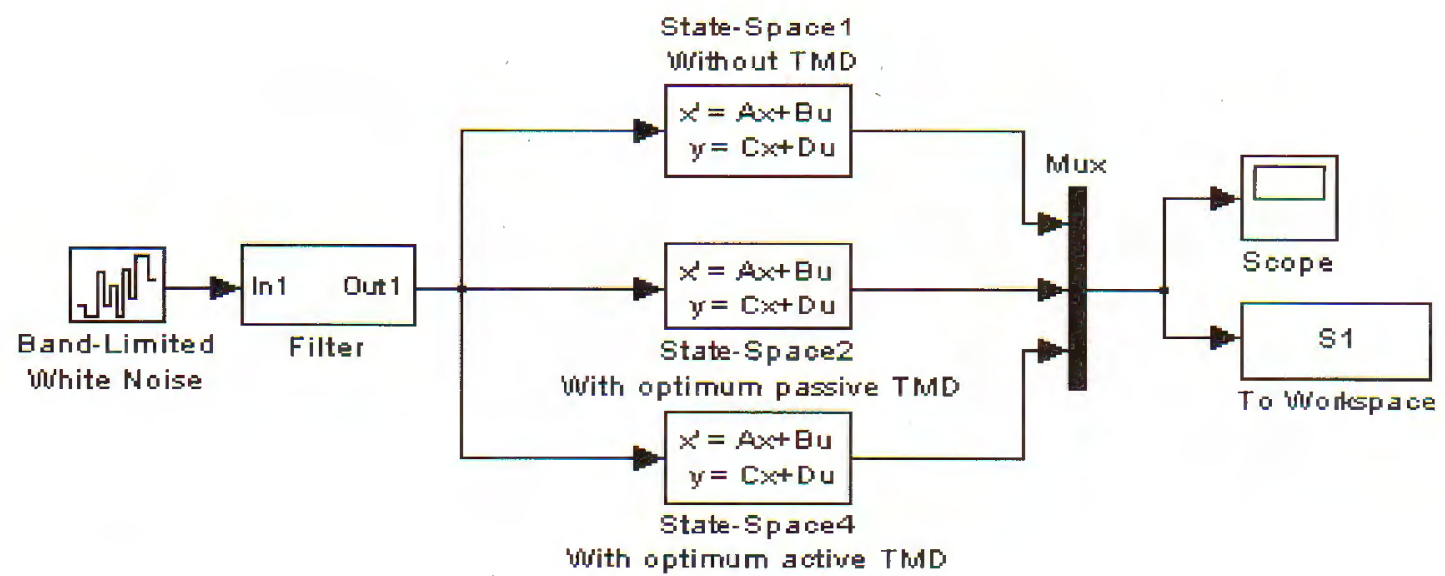

Fig. 3. Model Simulink 


\begin{tabular}{|c|c|c|c|c|c|}
\hline $\begin{array}{c}\text { Mean } \\
\text { squares }\end{array}$ & $\begin{array}{c}\text { Without } \\
\text { TMD }\end{array}$ & $\begin{array}{c}\text { Passive } \\
\text { TMD }\end{array}$ & $\begin{array}{c}\text { Active } \\
\text { TMD }\end{array}$ & $k_{p}$ & $k_{a}$ \\
\hline$\left\langle x_{1}^{2}\right\rangle$ & $0.4900 \mathrm{e}-9$ & $0.1700 \mathrm{e}-9$ & $0.10061 \mathrm{e}-9$ & 0.35 & 0.21 \\
\hline$\left\langle x_{2}^{2}\right\rangle$ & $0.14381 \mathrm{e}-8$ & $0.95402 \mathrm{e}-9$ & $0.20115 \mathrm{e}-9$ & 0.66 & 0.14 \\
\hline$\left\langle u^{2}\right\rangle$ & \multicolumn{5}{|c|}{$0.13889 \mathrm{e}-3$} \\
\cline { 1 - 3 }
\end{tabular}

Table. 1. Mean squares of $\left\langle x_{1}^{2}\right\rangle,\left\langle x_{2}^{2}\right\rangle,\left\langle u^{2}\right\rangle$ and $\left\langle p^{2}\right\rangle$

In order to illustrate the above calculated results, the vibration of the system is simulated using software SIMULINK in three cases: No TMD; Optimum passive TMD; Optimum active TMD. The simulink diagram is described in Fig. 3 and Fig. 4. The simulation diagrams are shown in Fig. 5 and Fig. 6.

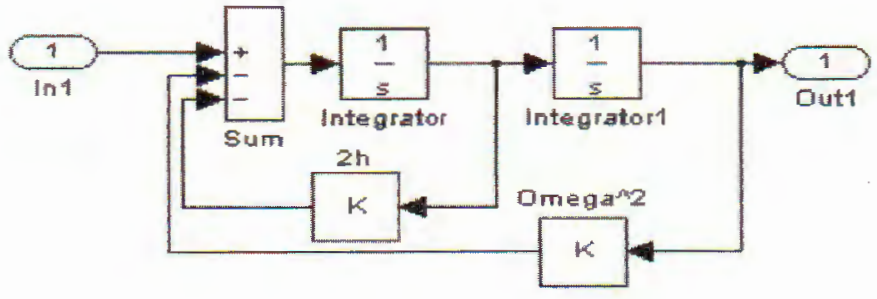

Fig. 4. Model Simulink-Filter

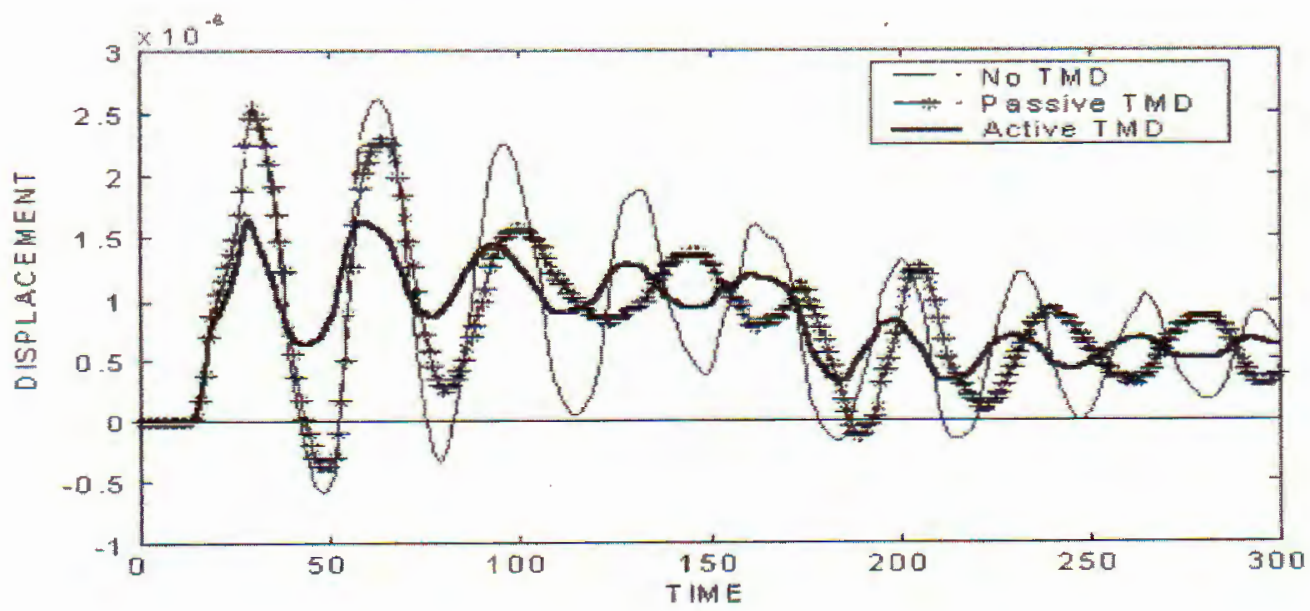

Fig. 5. Displacement of mass $m_{1}$ when system is without TMD, with optimum passive TMD and with optimum active TMD 


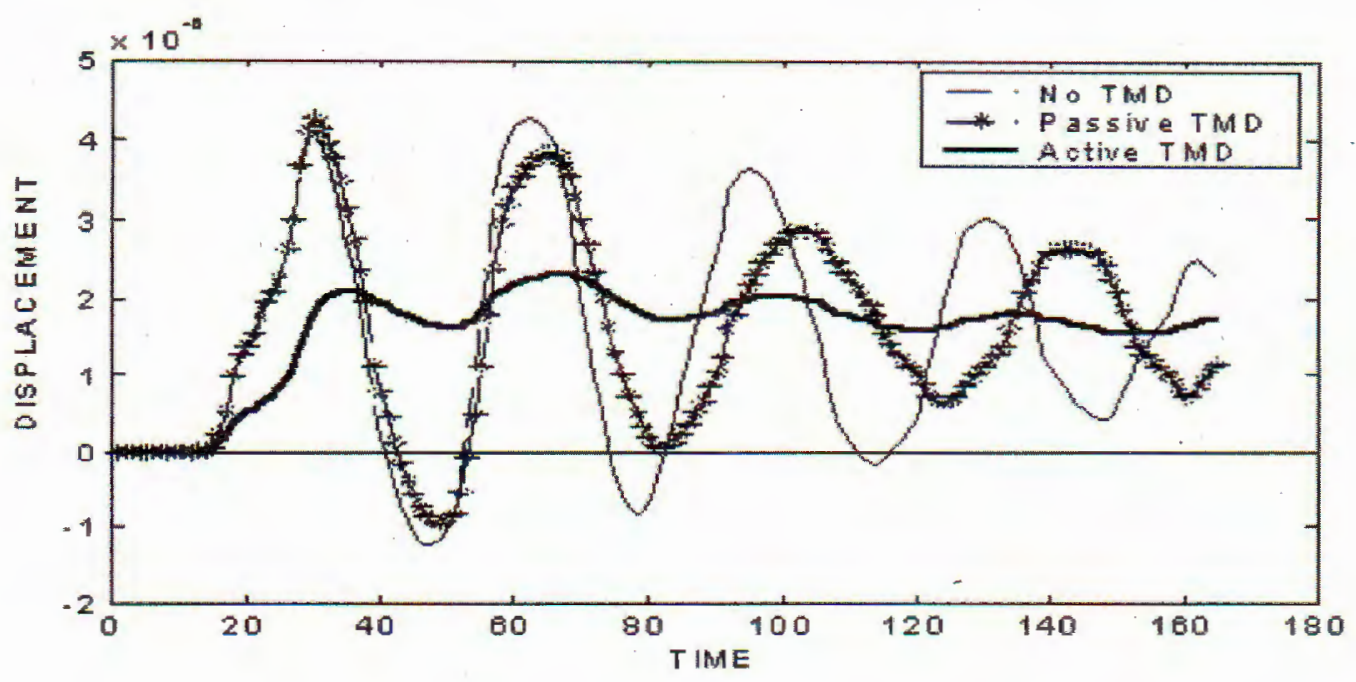

Fig. 6. Displacement of mass $m_{2}$ when system is without TMD, with optimum passive TMD and with optimum active TMD

From Fig. 5, Fig. 6, we can see that with optimum passive TMD, the displacements of mass $m_{i}(i=1(1) 2)$ are decreased approximately $40 \%$. However when optimum active TMD is used these displacements are decreased about $80 \%$. Therefore the application of active TMD here is better than the previous passive TMD [6].

\section{Conclusion}

To reduce undesired vibration which can cause damage to machines, vehicles, structures one can use passive or active TMD. The design of active TMD has attracted a great attentions in recent years because it can change the system energy by producing actuator force. The paper presents a design of active TMD for a multi-degree-of-freedom system subjected to base excitation which is modeled as a second coloured noise process. Upon feedback control theory, one can carry out the necessary conditions for the optimum control force. Then numerical calculations such as simulation and solving the moment equations are investigated to an example of 2-DOF system which applied the control force to a given passive TMD. The result show that the effective of active TMD is better than passive TMD. The result can be applied to other multi-degree-of-freedom systems subjected to different excitations.

Acknowledgement. The paper is supported by Vietnam Council for natural sciences.

\section{References}

1. Frahm H., Device for damping of bodies, US Patent No. 989, 958, 1911.

2. Den Hartog J. P., Mechanical Vibrations (3rd edn), McGraw - Hill: New York, 1947. 
3. Warburton G. B., Optimum absorber parameters for various combinations of response and excitation parameters, Earthquake Engineering and Structural Dynamics 10 (1982) 381 - 401.

4. Roberts J. B., Spanos P. D., Random Vibration and Stochastic Linearization, London, Wiley and Sons Ltd, West Sussex, England, 1990 .

5. Casciati F., Duc K. T. and Sang N. C., A selection of optimum parameters for tuned mass dampers, Proceedings of Third World Conference on Structural Control, Como, Italy 3 (2002) 753-758.

6. Nguyen Dong Anh, Nguyen Chi Sang, A selection of parameters of tuned mass damper for multi-degree-of-freedom systems subjected to second order coloured noise excitation, Vietnam Journal of Mechanics 25 (2003) 1-12.

7. Mehdi Setareh, Application of semi-active tuned mass dampers to base-excited systems, Earthquake Engineering and Structural Dynamics 30 (2001) 449-462.

8. Karnopp D. C., Active damping in road vehicle suspension systems, Vehicle System Dynamics 12 (1983) 291-316.

9. Chang J. C. H, Soong T. T., Structural control using active tuned mass dampers. Journal of the Engineering Mechanics Division, ASCE 106 (1980) 1091-1098.

10. Hrovat D., Barak P. and Rabins M., Semi-active versus passive or active tuned mass dampers for structural control, Journal of the Engineering Mechanics Division, ASCE 109 (1983) 691-705.

11. Abe M., Semi-active tuned mass dampers for seismic protection of civil structures, Earthquake Engineering and Structural Dynamics 25 (1996) 743-749.

Received May 14, 2003

\section{LỰC ĐIỀU KHIỄN TỐI UUU TÁC DỤNG LÊN TMD LẮP CHO HỆ NHIỀU BẬC TỰ DO}

Bài báo trình bày phương pháp thiết kế bộ TMD tích cực cho hệ nhiều bậc tự do dựa trên lý thuyết tối ưu bình phương tuyến tính. Đã xét tính toán số chi tiết cho hệ cơ học 2 bậc tự do. Kết quả cho thấy hiệu quả của bộ TMD tích cực tốt hơn bộ TMD thụ dộng. 